\title{
Controlled Studies in the Prehospital Setting: A Viable, Important Venue for Clinical Research
}

\author{
Paul E. Pepe, MD, FACEP
}

Since almost all life-threatening injuries and the great majority of cardiac arrests occur outside of the hospital and because care must be rendered within minutes to alter outcome, there would seem to be no better place to test early life-saving interventions than in the prehospital setting. ${ }^{1,2}$ Not only does the earliest intervention make a difference, but the prognosis for out-of-hospital arrest is far better than in-hospital..$^{3,4}$ However, the question arises as to whether scientific research actually can be accomplished successfully and reliably in the prehospital setting. The question relates to the fact that, in the United States, the actual prehospital emergency medical care is not provided by physician-scientists, but by persons who are physician surrogates (EMTs, paramedics). There exist some doubt that these EMS personnel can conduct reliable research using scientific methods. Moreover, the issue is complicated further by the question of patient consent in the prehospital setting.

Nevertheless, the capacity to conduct well-designed, prospective, controlled clinical studies in the prehospital setting has been demonstrated for cardiac arrest patients ${ }^{5,6}$ and for certain trauma interventions. ${ }^{7-11}$ For example, results of studies both with the pneumatic anti-shock garment (PASG) ${ }^{9-10}$ and infusions of $7.5 \%$ (hypertonic) saline/dextran $70^{11}$ have demonstrated the capability of evaluating the efficacy of these interventions with well-matched populations in the prehospital setting. ${ }^{10-12}$ Prospectively collected demographic and study/control group comparison data have validated the investigational designs. Thus, in these studies, one could confidently conclude that the only difference between the study and control groups was the single intervention being examined.

However, in accepting the fact that successful execution of well-controlled, prospective clinical studies using large patient populations is feasible in the prehospital setting, certain caveats must be acknowledged. The first qualification is that in those prehospital emergency medical care systems which have demonstrated the successful execution of such research, the physician-director of the EMS program essentially has clear medical control of the entire system. Furthermore, these respective medical directors share a common philosophy in which they regard the prehospital emergency care system to be part of their personal practice of medicine. ${ }^{13}$ Therefore, a key element in the systems that have effected sound clinical research is that the EMS medical director has the attitude that the standard of care given to patients by EMS personnel must be the best that he or she (the medical director) personally could have offered. ${ }^{1,13}$ In turn, this provides reasonable assurance that the paramedics under the medical director's supervision are competent and reliable, and that their performance will be

President, National Association of Emergency Medical Services Physicians (NAEMSP); Director, Emergency Medical Services, City of Houston, and Baylor College of Medicine. 
predictable. The results of such commitment are reflected in the remarkable survival statistics for critically ill and injured patients. ${ }^{2,5,10,14,17,18}$

The second caveat for success in prehospital EMS research is that all of the EMS system personnel involved (and the care delivered by them) are rigidly controlled; that is, this control of patient care extends into the hospital. ${ }^{2,18}$ This control is enhanced in certain locales where the EMS medical directors also are supervising faculty in the receiving facilities. By extending the control over protocol to include the care delivered in the hospital, better scientific control extends through the emergency department, operating suites, and the intensive care units. In turn, this further assures rigorous compliance with the research protocols.

A third caveat for success in prehospital research is the inclusion of the EMS personnel (who will be executing the protocol) in the early discussions regarding rationale, study design and methodology. In retrospect, this key element may be the feature missing in EMS systems that have experienced difficulties in protocol execution. ${ }^{15}$

A highly active, responsible EMS medical director actually creates a more controlled situation than is possible in most other clinical research settings. With a single, responsible physician in control of all medical procedures, the same standardized care is delivered by each of the paramedics under his or her direction, particularly if a strict protocol is effected..$^{1,11,18}$ On the other hand, in a hospital environment, one is more apt to find many physicians with an equal number of approaches, styles, and philosophies. As a result, protocols often are modified by such independent spirits. In contrast, in a paramilitary EMS organization (under intensive, singular medical direction), there exist better guarantees of rigid, standardized, systematic implementation of study protocols. Again, this rigid standardization helps to assure that the intervention under investigation is the only factor separating a study group from a control group. ${ }^{9,11}$

There are other factors that make the prehospital setting an excellent venue for the performance of clinical investigations. Beyond the exceptional occupational enthusiasm of EMS personnel, one usually finds in the urban North American paramedic, a specialized expertise for dealing quickly and effectively with cardiac and trauma resuscitation. This expertise is reinforced by the highvolume of cases that they encounter. Compared to the average emergency department physician, they see, more regularly, patients requiring resuscitation and these specific skills are honed further by regularly scheduled performance reviews and retraining, as well as by the constant feedback of a street-wise, intensely involved medical director. ${ }^{1,13}$ In essence, paramedics can follow resuscitation protocols closely because they specifically do that for a living. And when they do it every day, they do it well in supervised systems.

One more factor that enhances the feasibility of scientific research in the prehospital setting is the large number of patients that can be studied. Large study populations are possible because the entire catchment of the whole EMS system potentially is available. It is less likely that a statistically desirable number of resuscitation patients can be entered into a study from a single hospital emergency department. The longer the period over which a study is conducted, the more likely it is to be affected by other factors.

Since investigational studies classically have originated as an inhospital phenomenon, there are some philosophical and logistical problems that have to be considered for prehospital studies. These hurdles 
include: 1) seeking permission to bring study patients to and continue the protocol at various receiving hospitals; 2) developing deferred consent procedures; and 3) assuring the paramedics that they will be protected from liability. In highly complex or highly experimental interventions (e.g., the prehospital use of thrombolytic therapy by paramedics), some of these problems can be addressed by limiting the number of EMS personnel that actually carry out the protocol, at least initially. For example, in the Houston system, the three senior EMS supervisors, who oversee each of the three different 24-hour shifts of paramedics, often are used initially in resuscitation research using investigational drugs. They drive their own response vehicles and usually are available to respond to all cardiac arrests which occur in the prehospital setting, ${ }^{16}$ usually an average of 25 cardiac arrests per senior supervisor per month. Prior to any project, detailed instructions and training are given to these three senior paramedics whose job it may be to initiate administration of the experimental therapy in the field. These instructions usually involve study entry and exclusion criteria as well as the background information relevant to the study. However, for the most part, because they usually help in study design, instruction is relatively easy. In addition, better care and stricter protocol adherence has been rendered during any protocol because paramedic supervisors arrive at practically every arrest in the city (3-4 per day). Furthermore, because they are under extremely close scrutiny by physicians who specialize in prehospital care and cardiac arrest management, these paramedics' skills, judgment, and performance in such cases generally exceed that of most physicians.

A major proviso in the willingness of paramedics to carry out studies is the reliability and quality of on-line physician involvement in the event of an unanticipated problem. ${ }^{16}$ Physicians who regularly provide supervision to the paramedics, and are the usual resource for the paramedics in case of complications best suit this purpose.

In terms of deferred consent and receiving hospital approval, the case can be presented to institutional review boards that the overwhelming majority of patients who have major injury or who have cardiac arrest due to primary cardiac disease, are dependent on care rendered in the prehospital setting. ${ }^{18,19}$ If some timedependent therapies are initiated only after the patient arrives at the hospital, fewer study patients would benefit from it (let alone survive). Paramedics can be very capable in cardiac resuscitation and there could be no better time to administer a new interventional drug than "immediately." And what is the alternative? A proposed complication seems to be inconsequential in someone who has no other option but death. As long as the study is carefully designed, carefully executed, and carefully monitored and scrutinized for safety, true complications can be addressed accordingly.

In summary, the prehospital setting provides an excellent venue for the execution of prospective, controlled clinical trials. This venue offers a potential for the implementation of excellent, standardized research protocols and a large patient base from which to enroll study participants. Moreover, it provides an arena in which early therapeutic care has the best potential to significantly improve the outcome for many people. With thousands and thousands of injuries that may be amenable to some new therapy, and nearly 250,000 potentially treatable cases of sudden death occurring each year, in the United States alone, the obvious magnitude and impact of 
even a small improvement in care will be profound.

\section{References}

1. Pepe PE, Stewart RD: Role of the physician in the prehospital setting. Ann Emerg Med 1986;15:1480-1483.

2. Pepe PE, Stewart RD, Copass MK: Prehospital management of trauma. Ann Emerg Med 1986;15:1484-1489.

3. Bonnin MJ, Pepe PE, Clark PS: Survival prognosis for the elderly following out-ofhospital cardiac arrest. Ann Emerg Med 1989;18:469.

4. Taffet GE, Teasdale TA, Luchi RJ: Inhospital cardiopulmonary resuscitation. JAMA 1981;260:2069-2072.

5. Weaver WD, Cobb LA, Copass MK, Hallstrom AP: Ventricular fibrillation: A comparative trial using $175-\mathrm{J}$ and $320-\mathrm{J}$ shocks. NEnglJ Med 1982;307:1101-1106.

6. Haynes RE, Chinn TL, Copass MK, Cobb LA: Comparison of bretylium tosylate and lidocaine in management of out-of-hospital ventricular fibrillation: A randomized clinical trial. Am J Cardiol 1981;48:353-356.

7. Mattox KL, Bickell WH, Pepe PE, Mangelsdorff AD: Prospective randomized evaluation of antishock MAST in posttraumatic hypotension. $J$ Trauma 1986;26:779-784.

8. Pepe PE, Bickell WH, Mattox KL: The effect of anti-shock garments on prehospital survival: The need for controlled clinical studies. Prehospital and Disaster Medicine 1987;3:40-45.

9. Pepe PE, Bass RR, Mattox KL: Clinical trials of the pneumatic antishock garment in the urban prehospital setting. Ann Emerg Med 1986;15:1407-1410.

10. Bickell WH, Pepe PE, Wyatt $\mathrm{CH}$, et al: The effect of antishock garments on patients with penetrating abdominal injuries. Ann Emerg Med 1987;16:653-658.

11. Mattox KL, Maningas PA, Pepe PE, et al: Hypertonic saline/dextran 70 in prehospital management of post-traumatic hypotension: Preliminary observations. Am J Surgery 1989;157:528-534.

12. Personal Correspondence from Department of Army, Division of Military Trauma Research, Col. Edwin S. Beatrice, Commander, U.S. Army Institute of Research, Division of Military Trauma Research, San Francisco, California, USA, December 15, 1986.

13. Pepe PE, Bonnin MJ: Limitations and liabilities. Emergency 1989;2140-2143.

14. Pepe PE, Mattox KL, Prentice FD. Impact of full-time physician supervision on an urban EMS system. Prehosp. Disaster Med. 1989;5:7.

15. Heller MB, Melton JB, Kaplan RM, et al: Data collection by paramedics for prehospital research. Ann Emerg Med 1988; 17:414415.

16. Emergency Medical Services in the City of Houston. Publication of the City of Houston Fire Department, 1989 Houston, Texas, USA.

17. Copass MK, Oreskovich MR, Bladergroen MR, Carrico CJ. Prehospital cardiopulmonary resuscitation of the critically injured patient. Am J Surg. 1984;148:20-26.

18. Pepe PE, Copass MK. Prehospital care. In: Early Care of the Injured. American College of Surgeons Committee on Trauma. EE Moore (Ed). B.C. Decker, Inc. Philadelphia, 4th Edition. 1990;37-55.

19. Bonnin MJ, Pepe PE, Clark PS: Key role of prehospital resuscitation in survival from out-of-hospital cardiac arrest. Ann Emerg Med. 1990;19:466. 